\title{
Expressão de Proteína-1 Relacionada a Receptor de Lipoproteína de Baixa Densidade (LRP1) em Monócito em Correlação com EIMC em Pacientes Mexicanos Hipertensos
}

\author{
Monocyte Low-Density Lipoprotein Receptor-Related Protein 1 (LRP1) Expression Correlates with cIMT in \\ Mexican Hypertensive Patients
}

Ricardo Gamboa, ${ }^{1}$ María José Jaramillo-Estrella, ${ }^{1}$ María del Rocio Martínez-Alvarado, ${ }^{1}$ Maria Elena Soto, ${ }^{1}$ Yazmin Estela Torres-Paz, ${ }^{1}$ David de Gonzalo-Calvo, ${ }^{2}$ Leonardo Del Valle-Mondragón, ${ }^{1}$ Rebeca López-Marure, ${ }^{1}$ Vicenta C. Llorente-Cortés, ${ }^{2}$ Claudia Huesca-Gómez ${ }^{1}$

Instituto Nacional de Cardiologia Ignacio Chavez, ${ }^{1}$ Ciudad de México - México

Hospital de Sant Pau - Lipids and Cardiovascular Pathology Group, ${ }^{2}$ Barcelona, Catalunya - Espanha

\section{Resumo}

Fundamento: A hipertensão arterial (HTA) representa um grande fator de risco de morbidade e mortalidade cardiovascular. Ainda não se sabe que mecanismos moleculares específicos estão associados ao desenvolvimento de hipertensão essencial.

Objetivo: Neste trabalho, analisamos a associação entre expressão mRNA de monócito LRP1, expressão de proteína LRP1, e espessura íntima-média de carótida (EIMC) de pacientes com hipertensão essencial.

Métodos: A expressão mRNA de monócito LRP1 e os níveis de proteína e EIMC foram quantificados em 200 indivíduos mexicanos, sendo 91 normotensos (NT) e 109 hipertensos (HT) A significância estatística foi definida em p $<0,05$.

Resultados: O grupo de pacientes HT tinha EIMC maior altamente significativa em comparação com os pacientes NT $(p=0,002)$, e isso está relacionado ao aumento na expressão mRNA de LRP1 $(6,54$ versus. 2,87$)(p=0,002)$ e expressão de proteína LRP1 $(17,83$ versus 6,25$)$, respectivamente $(p=0,001)$. Essas diferenças foram mantidas mesmo quando dividimos nossos grupos de estudo, levando em consideração apenas aqueles que apresentavam dislipidemia na expressão de mRNA $(p=0,041)$ e de proteínas $(p<0,001)$. Também se identificou que a indução de LRP1 mediada por LRP1 em monócitos em de maneira dependente de dose e tempo, com diferença significativa em NT versus HT $(0,195 \pm 0,09$ versus $0,226 \pm 0,12, p=0,046)$.

Conclusão: Foi encontrado um aumento em EIMC em indivíduos com hipertensão, associada a expressões de proteína LRP1 e mRNA mais altas em monócitos, independente da presença de dislipidemia em pacientes HT. Esses resultados que a upregulation de LRP1 em monócitos de pacientes hipertensos mexicanos poderia estar envolvida na diminuição da EIMC. (Arq Bras Cardiol. 2021; 116(1):56-65)

Palavras-chave: Monócitos; LRP1; mRNA; Hipertensão/epidemiologia, México; Espessura Intima Média Carótida.

\footnotetext{
Abstract

Background: Arterial hypertension (HT) represents a major risk factor for cardiovascular morbidity and mortality. It is not yet known which specific molecular mechanisms are associated with the development of essential hypertension
}

Objective: In this study, we analyzed the association between LRP1 monocyte mRNA expression, LRP1 protein expression, and carotid intima media thickness (CIMT) of patients with essential hypertension.

Methods: The LRP1 monocyte mRNA expression and protein levels and cIMT were quantified in 200 Mexican subjects, 91 normotensive (NT) and 109 hypertensive (HT). Statistical significance was defined as $p<0.05$.

Correspondência: Claudia Huesca-Gómez •

Instituto Nacional de Cardiologia Ignacio Chavez - Physiology - “. Juan Badiano No 1, Col. Sección XVI Mexico 14080 - México

E-mail: claudia.huesca@cardiologia.org.mx

Artigo recebido em 09/08/2019, revisado em 07/02/2020, aceito em 16/03/2020

DOI: https://doi.org/10.36660/abc.20190535 
Results: HT patients group had highly significant greater cIMT as compared to NT patients $(p=0.002)$ and this correlated with an increase in the expression of LRP1 mRNA expression (6.54 vs. 2.87) ( $p=0.002)$ and LRP1 protein expression (17.83 vs. 6.25), respectively $(p=0.0001)$. These differences were maintained even when we divided our study groups, taking into account only those who presented dyslipidemia in both, mRNA ( $p=0.041)$ and proteins expression $(p<0.001)$. It was also found that Ang II mediated LRP1 induction on monocytes in a dose and time dependent manner with significant difference in NT vs. HT (0.195 \pm 0.09 vs. $0.226 \pm 0.12, p=0.046)$.

Conclusion: An increase in CIMT was found in subjects with hypertension, associated with higher mRNA and LRP1 protein expressions in monocytes, irrespective of the presence of dyslipidemias in HT patients. These results suggest that LRP1 upregulation in monocytes from Mexican hypertensive patients could be involved in the increased cIMT. (Arq Bras Cardiol. 2021; 116(1):56-65)

Keywords: Monocytes; LRP1; mRNA; EIMC; Hypertension/epidemiology; Mexico; Carotid Intima Media Thickness.

Full texts in English - http://www.arquivosonline.com.br

\section{Introdução}

A hipertensão arterial (HTA) é uma doença crônica e multifatorial que constitui um problema grave de saúde pública. ${ }^{1} \mathrm{~A}$ hipertensão raramente causa sintomas em estágios iniciais; é uma assassina silenciosa, causando aterosclerose acelerada, dano a órgãos importantes, incapacidade e morte por doenças cardiovasculares. ${ }^{2}$

Lesões ateroscleróticas incluem células endoteliais alteradas, monócitos circulantes, migração de células musculares lisas vasculares (CMLV), e desenvolvimento de células espumosas. ${ }^{3} \mathrm{O}$ endotélio alterado permite a entrada e retenção de lipoproteína de baixa densidade (LDL) na camada íntima. ${ }^{4}$ Quando o LDL está preso na íntima arterial, ele passa por alterações tais como oxidação e agregação, o que facilita a captação por monócitos-macrófagos da íntima e (CMLV), por meio de seu reconhecimento por receptores de LDL não clássicos. ${ }^{5}$ Esses receptores não são regulados por colesterol e permitem uma captação em massa de LDL modificado que causa acúmulo lipídico intracelular.

A proteína-1 relacionada a receptor de lipoproteína de baixa densidade (LRP1), que é uma proteína transmembrana multiligante $^{6}$ pertencente à família LDLR. Ela é expressa em diferentes células semelhantes a neurônios, fibroblastos, células tumorais, hepatócitos, células musculares lisas vasculares, e monócitos e macrófagos. ${ }^{7,8}$ Sabe-se que ela participa na captação do LDL modificado ${ }^{9} \mathrm{e}$ é tem expressão excessiva em placas ateroscleróticas em modelos animais e humano. ${ }^{10,11}$

Além disso, a expressão de genes de LRP1 é aumentada em células mononucleares de pacientes com obstrução coronária. ${ }^{12,13}$ Em monócitos e macrófagos, a LRP1 contribui para a captação do LDL modificado agregado. ${ }^{14,15}$ Entretanto, os efeitos da hipertensão na expressão de LRP1 em humanos não são exatamente conhecidos. Portanto, a obtenção de monócitos circulantes possibilitou o estudo dos mecanismos de sua participação na formação de placa aterosclerótica. ${ }^{16} \mathrm{De}$ outra forma, o EIMC é considerado um excelente marcador não invasivo de doença cardiovascular, e foi associado a aterosclerose e fatores de risco cardiovascular ${ }^{17,18}$ e à prevalência de doença cardiovascular, provando que é útil no diagnóstico de aterosclerose. ${ }^{19-21}$ Conforme mencionado acima, o objetivo deste trabalho era o estudo de níveis de LRP1 e mRNA e de expressão de proteína e monócitos de pacientes com hipertensão arterial essencial, e sua correlação com a espessura íntima-média de carótida.

\section{Métodos}

\section{População e Modelo do Estudo}

Um total de 200 indivíduos mexicanos não relacionados (109 pacientes diagnosticados com hipertensão, e 91 indivíduos normotensos) foram recrutados no Instituto Nacional de Cardiologia "Ignacio Chávez". Os critérios de inclusão de ambos os grupos eram: terem nascido no México e ser descendente de pelo menos 3 gerações anteriores, ter mais de 40 anos de idade, e concordar em participar do estudo, assinando o consentimento informado. Os controles eram indivíduos aparentemente saudáveis, assintomáticos, sem histórico familiar de hipertensão ou doença cardiovascular prematura, com pressão sanguínea $\leq 120 / 80$ mmHg. Para o grupo de hipertensos, os indivíduos tinham pressão sanguínea $\geq 140 / 90 \mathrm{mmHg}$ ou já tinham sido diagnosticados anteriormente com hipertensão essencial. O critério de exclusão foi sofrer de uma doença degenerativa crônica. Todos os participantes responderam a questionários padronizados e validados para obter informações sobre seu histórico familiar e médico, consumo de álcool e tabagismo, hábitos alimentares, e atividade física.

O comitê de ética do Instituto Nacional de Cardiologia "Ignacio Chávez" aprovou o projeto. Os pacientes preencheram formulários de consentimento informado antes do estudo. Todos os procedimentos estavam de acordo com a Declaração de Helsinki de 1975, conforme sua revisão de 2013.

\section{Medições Antropométricas}

Os indivíduos selecionados passaram por medições antropométricas para determinar suas alturas em metros (m) e seus pesos em quilogramas $(\mathrm{kg})$. A pressão sanguínea foi medida utilizando-se um esfigmomanômetro de mercúrio, em conformidade com as recomendações do VII Comitê Nacional Conjunto para Prevenção, Detecção, Avaliação, e Tratamento de Pressão Sanguínea Alta (JNC VII).

\section{Espessura da Íntima-média da Artéria Carótida}

Um especialista em resolução de ultrassonografia avaliou a espessura íntima-média de carótida (EIMC). Todas as medições foram realizadas com o ultrassom A Sonosite Micromax acoplado a um transdutor linear de alta resolução e multifrequência. As medições foram feitas na carótida comum após exame de uma seção longitudinal de $10 \mathrm{~mm}$ a $2 \mathrm{~cm}$ de distância da bifurcação. A parede anterior ou proximal e 
na parede posterior ou distal foram medidas nas projeções lateral, anterior e posterior, seguidas de um eixo perpendicular à artéria, para discriminar duas linhas: uma para a interface íntima-sangue, e outra na interface média-adventício. Cinco medições foram obtidas da carótida direita, e 5 da carótida esquerda, usando valores médios (EIMC média) e máximos (EIMC máxima), automaticamente calculados pelo software. A EIMC foi considerada anormal com valores maiores ou iguais ao 75 percentil por idade e sexo. ${ }^{22}$

\section{Determinações Bioquímicas}

Foram coletadas amostras de sangue após 12 horas de jejum. Foram medidos: glicemia, colesterol total (CT), triglicérides (TG), e colesterol de lipoproteína de alta densidade (HDL-C) em amostras frescas (plasma em jejum), usando procedimentos enzimáticos padronizados em um analisador Hitachi 902 (Hitachi Ltd, Tóquio, Japão); o colesterol de lipoproteína de baixa densidade (LDL-C) foi estimada utilizando-se a fórmula de DeLong et al. ${ }^{23}$ Todos os testes passaram por um esquema de controle de qualidade externo (Lipid Standardization Program, Center for Disease Control em Atlanta, GA, EUA).

As concentrações de soro Ang II foram avaliadas por eletroforese na zona capitar conforme descrito anteriormente. ${ }^{24}$ Os níveis totais de proteína $C$ reativa de alta sensibilidade total (PCR-as) foram determinados pela imunonefelometria em um nefelômetro BN Pro Spec (Dade Behring Marburg $\mathrm{GmbH}$, Alemanha). Os valores de variação de coeficiente (VC) entre ensaios foram $<6 \%$ para todos esses ensaios. O colesterol não HDL (não HDL-C) foi calculado subtraindo-se o HDL-C do colesterol total. O valor de dislipidemia foi definido de acordo com fatores de risco cardiovascular convencionais: (TC) $\geq 200 \mathrm{mg} / \mathrm{dL}$ e/ou HDL-C $\leq 40 \mathrm{mg} / \mathrm{dL} /$ ou LDL-C $\geq 130$ $\mathrm{mg} / \mathrm{dL}$ e/ou TG $\geq 150 \mathrm{mg} / \mathrm{dL}$.

\section{Separação de Monócito de Sangue Periférico}

O sangue integral coletado em tubos com EDTA foi diluído 1:1 com heparina PBS $1 \times-1 \%$; em seguida, foi adicionado Histopaque 1077 (10771, Sigma-Aldrich). As células sanguíneas mononucleares periféricas (CSMP) foram obtidas da faixa branca central do gradiente após a centrifugação. Em seguida, os monócitos foram obtidos pelo enriquecimento direto das células CD14+ pelo sistema de classificação magnética (MACS; Miltenyi Biotec, Bergisch-Gladbach, Alemanha). Uma alíquota de $1 \mathrm{~mL}$ de reagente Tripure ${ }^{\mathrm{TM}}$ (Roche Molecular Biochemicals) foi adicionada para coletar os monócitos. As células foram armazenadas a $-80{ }^{\circ} \mathrm{C}$.

\section{Linha Celular de Cultura THP-I}

Células de leucemia monocítica humana foram mantidas em uma cultura em suspensão de meio RPMI-1640 (GibcoBRL) contendo $2 \mathrm{mM}$ de glutamina, $25 \mathrm{mM}$ HEPES, 1,5 g/L de bicarbonato de sódio, $50 \mathrm{U} / \mathrm{mL}$ de penicilina, e $50 \mu \mathrm{g} / \mathrm{mL}$ de estreptomicina (Sigma), suplementados com $10 \%$ soro fetal bovino (SFB), a $37^{\circ} \mathrm{C}$, em $5 \% \mathrm{CO} 2$. Células THP-1 paradas foram pré-incubadas com Ang II $(1 \mu \mathrm{mol} / \mathrm{L})$ para aumentar os períodos de análise dos efeitos da Ang II na expressão de LRP1 nos monócitos. A dose de angiotensina II foi selecionada com base em estudos prévios em nosso grupo e oferece uma concentração de plasma de angiotensina II de forma semelhante ao reportado em pacientes com hipertensão. ${ }^{25}$

\section{Extração de RNA e Síntese de cDNA}

O RNA total foi extraído utilizando o Reagente de isolamento Tripure ${ }^{\mathrm{TM}}$ (Roche Molecular Diagnostics, Indianápolis, EUA), de acordo com as instruções do fabricante. O rendimento e a qualidade do RNA foram avaliados com eletroforese de gel de agarose 1\%; RNA foi armazenado a $-80{ }^{\circ} \mathrm{C}$ até serem analisadas. A reação de transcrição reversa foi realizada utilizando-se $1 \mu \mathrm{g}$ do RNA total para síntese de cDNA de acordo com o kit High Capacity cDNA Reverse Transcription kit (Applied Biosystems Foster City, CA, EUA). $\mathrm{O}$ cDNA foi armazenado a $-80^{\circ} \mathrm{C}$.

\section{Ensaios de Expressão Gênica}

A expressão gênica de LRP1 (Hs00233899_m1) eHPRT (Hs99999909_m1) (gene endógeno) foram realizádas via uma reação em cadeia de polimerase de transcrição reversa em tempo real e semiquantitativa (RT-PCR), utilizando-se um kit comercial. A "Expressão Gênica TaqMan" foi realizada utilizando-se $1 \mu \mathrm{l}$ de produtos de transcrição reversa misturados com $10 \mu \mathrm{l}$ de TaqMan Universal PCR Master Mix (Applied Biosystems, Foster City, CA, EUA), $1 \mu \mathrm{l}$ 20x ensaios em $8 \mu \mathrm{l}$ de água sem nuclease. Depois de misturar ligeiramente, a mistura foi transferida para uma microplaca de PCR de tempo real, utilizando o equipamento 7300 Real Time PCR System (Applied Biosystems).

As condições usadas foram: $50^{\circ} \mathrm{C}$ por 2 min e $10 \mathrm{~min}$ a $95^{\circ} \mathrm{C}$, seguido de 40 ciclos a $95^{\circ} \mathrm{C}$ por $15 \mathrm{~s}$, e $60^{\circ} \mathrm{C}$ por $1 \mathrm{~min}$. Os níveis de expressão foram medidos em duplicata e os valores de ciclo de limiar [Ct] foram determinados e normalizados usando a expressão gênica endógena (HPRT).

\section{Análise Western Blot}

A proteína total foi isolada de monócitos, utilizandose o Reagente de isolamento TriPure ${ }^{\mathrm{TM}}$ (Roche Molecular Diagnostics, Indianápolis, EUA), de acordo com as instruções do fabricante. A proteína foi quantificada utilizando-se o Ensaio proteico Pierce BCA (Thermo Scientific, Waltham, MA, EUA). Valores equivalentes de proteína total $(25 \mu \mathrm{g})$ foram carregados em gel SDS-poliacrilamida a $10 \%$ (v/v) sob condições de redução. As amostras foram eletrotransferidas para membranas de nitrocelulose, que estavam saturadas em temperatura ambiente $1 \mathrm{~h}$ em TTBS (20 mM Tris- $\mathrm{HCl}$, pH 7,5, $500 \mathrm{mM} \mathrm{NaCl}, 0,01 \%$ Tween 20 e 5\% de leite desnatado). Foram realizadas análises de western blot utilizando anticorpos monoclonais específicos contra a LRP1 humana (85kDa -chain, clone 8B8 RDI 61067, diluição1:40) e os anticorpos secundários correspondentes (1:10.000 diluição; Dako; Glostrup, Dinamarca). O software QuantityOne (Bio-Rad, Hercules, CA, EUA) foi usado para quantificar as faixas presentes nas membranas via densitometria, e foram detectados utilizando o reagente de detecção ECL Prime Western Blotting (Amersham). Os níveis de expressão foram medidos em duplicata e normalizados pela comparação com a concentração de controle de proteína de carga. Os resultados foram expressos como unidades de intensidade arbitrária. 


\section{Análise Estatística}

Os dados foram analisados usando o software SPSS v19 (SPSS Inc. Chicago EUA). Os resultados foram expressos como média \pm desvio padrão (DP) em variáveis contínuase as variáveis categóricas foram expressas em porcentagem. O teste usado para avaliar a normalidade foi o Shapiro-Wilk. A comparação entre grupos foi realizada usando o teste t Student não pareado para variáveis contínuas e teste qui-quadrado para variáveis categóricas. A análise de correlação foi feita de acordo como método de Pearson. Regressões logísticas múltiplas foram usadas para explorar as associações entre a expressão de EIMC e LRP1. Os dados são apresentados como razão de chance (RC), com um intervalo de confiança de $95 \%$. Um valor de $p<0,05$ foi considerado estatisticamente significativo. O tamanho da amostra foi calculado usando a referência de Schulz 2002. ${ }^{13}$ De acordo com proporções de amostras independentes levando em consideração uma incidência de gene de LRP1 de aproximadamente 0,08 nos casos e 0,02 nos controles, com um $\Delta=0,06$, e um poder estatístico de 95\%, p <0,05. De acordo com a seguinte fórmula, nosso valor de $\mathrm{n}$ foi $=70$.

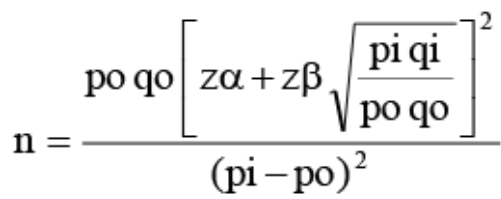

po $=$ Probabilidade de que a expressão de LRP1 ocorra em casos

$\mathrm{q} 0=$ Probabilidade de que a expressão de LRP1 não ocorra em casos

$\mathrm{pi}=$ Probabilidade de que a expressão de LRP1 ocorra em controles

qi = Probabilidade de que a expressão de LRP1 não ocorra em controles

$$
1,96=\text { valor }<0,05
$$$$
1,28=\text { poder }(0,84)
$$

$$
n=\frac{(0.8)(0.92)\left[1.96+1.28 \sqrt{\frac{p(0.02) q(0.98)}{(0.08)(0.92)}}\right]^{2}}{((0.08)-(0.02))^{2}}
$$

\section{Resultados}

\section{Características da População do Estudo}

Uma população de 200 indivíduos mexicanos foi estudada, sendo que 91 eram indivíduos normotensos (NT), e 109 eram hipertensos (HT). As características bioquímicas e antropométricas da população estudada são mostradas na Tabela 1. Da população total, 62,5\% eram mulheres, e 37,5\% eram homens. Idade, índice de massa corporal (IMC), EIMC, HDL-C, proteína C reativa, Ang II, e índices de LDL-C/HDL-C, TC/HDL-C, TG/HDL-C apresentaram estatísticas diferentes entre os grupos. Esses parâmetros foram mais altos no grupo dos hipertensos, em comparação com o grupo normotenso, exceto para os níveis de $\mathrm{HDL}-\mathrm{C}$, que foram mais baixos no grupo dos hipertensos. A prevalência de obesidade foi $19,8 \%$ no grupo dos normotensos e $44,1 \%$ in no grupo dos hipertensos. Quando foi feita a comparação entre ambos os sexos, com os mesmos parâmetros, não foram encontradas diferenças significativas. Também comparamos nossos grupos de acordo com níveis de dislipidemia conforme ATP III. Entretanto, houve apenas diferenças significativas em HDL-C $(\leq 40 \mathrm{mg} / \mathrm{dL}),(\mathrm{NT}=16,5 \%$ versus $\mathrm{HT}=32,7 \%, \mathrm{p}=0,001) \mathrm{e}$ triglicérides $(\geq 150 \mathrm{mg} / \mathrm{dL})(\mathrm{NT}=42,7 \%$ versus $\mathrm{HT}=57,3 \%$, $\mathrm{p}=0,001)$ (dados não mostrados).

\section{Correlação entre Hipertensão e Expressão de LRP1 de Monócitos.}

Com o objetivo de saber os níveis de mRNA e expressão de proteína, foram realizadas análises em LRP1 para ambos os grupos (Figura 1). Foram encontradas diferenças significativas entre os grupos de NT e HT na expressão de mRNA $(p=0,002)$ e expressão de proteína $(p=0,001)$. Portanto, quando os indivíduos foram comparados em grupos de homens e mulheres, uma diferença significativa só foi encontrar em LRP1 mRNA em nos indivíduos hipertensos. Houve uma expressão excessiva no grupo das mulheres em comparação com os homens $(p=0,044)$. Além disso, encontramos um aumento na expressão de LRP1 mRNA e proteína em indivíduos dislipidêmicos hipertensos, em comparação com sujeitos dislipidêmicos normotensos (dados não exibidos.

Por outro lado, para examinar se outros fatores, como EIMC e Ang II poderiam participar dos valores de pressão sanguínea, analisamos essas variáveis (Tabela 2). Foi encontrada uma diferença significativa entre os grupos NT e HT em relação a EIMC $(p=0,002)$ e Ang II $(p=0,046)$, respectivamente. Entretanto, quando dividimos o grupo de acordo com o sexo, não encontramos diferenças em nenhum dos parâmetros estudados.

\section{Efeitos da Angiotensina II nos Níveis de Expressão do Monócito LRP1}

Para estudar os efeitos da indução de LRP1 mediada por Ang II nos monócitos, a linha celular do monócito THP1 foi incubada com Ang II por 4h e 8h, com concentrações de 1 e $10 \mu \mathrm{M}$. Na linha celular do monócito THP1, a Ang II aumentou a expressão de LPR1 mRNA de maneira dependente de dose e tempo, sendo mais evidente após 8 horas de incubação (Figura 2).

\section{Associação entre Expressão de LRP1 Monócitos e Espessura Íntima/média da Carótida de Pacientes com Hipertensão.}

Para saber se havia uma relação entre a espessura da EIMC e expressão de LRP1 mRNA e/ou expressão de proteína LRP1, foram realizadas regressões logísticas múltiplas ajustadas por perfil lipídico, idade e sexo (Tabela 3). Uma diferença significativa foi encontrada entre EIMC e os níveis de expressão de LRP1 mRNA $(p=0,047)$ e os níveis de proteína LRP1 $(p=0,039)$ em pacientes hipertensos. 


\section{Artigo Original}

Tabela 1 - Características antropométricas, clínicas e bioquímicas dos pacientes do estudo

\begin{tabular}{|c|c|c|c|}
\hline Parâmetros & $\begin{array}{l}\text { Normotensos } \\
\quad(\mathrm{N}=91)\end{array}$ & $\begin{array}{l}\text { Hipertensos } \\
\quad(\mathrm{N}=109)\end{array}$ & $p$ \\
\hline Idade (anos) & $46,0 \pm 11,35$ & $50,36 \pm 11,57$ & 0,007 \\
\hline $\operatorname{Sexo}(F / M)(\%)$ & $61,5 / 37,5$ & $64 / 36$ & 0,313 \\
\hline Peso (kg) & $71,44 \pm 14,30$ & $75,21 \pm 12,71$ & 0,056 \\
\hline Altura $(\mathrm{cm})$ & $161,99 \pm 9,81$ & $159,39 \pm 9,06$ & 0,057 \\
\hline $\mathrm{BMI}\left(\mathrm{Kg} / \mathrm{m}^{2}\right)$ & $26,92 \pm 4,06$ & $29,36 \pm 3,77$ & $<0,001$ \\
\hline PSS (mmHg) & $110,23 \pm 9,07$ & $142,78 \pm 10,82$ & $<0,001$ \\
\hline DPS (mmHg) & $69,90 \pm 75,85$ & $91,94 \pm 7,72$ & $<0,001$ \\
\hline $\operatorname{EIMC}(\mathrm{mm})$ & $0,587 \pm 0,16$ & $0,729 \pm 0,16$ & 0,002 \\
\hline EIMC máx. (mm) & $0,606 \pm 0,18$ & $0,787 \pm 0,16$ & 0,008 \\
\hline Colesterol total (mg/dL) & $197,32 \pm 40,41$ & $198,91 \pm 37,42$ & 0,772 \\
\hline Triglicérides (mg/dL) & $166,56 \pm 94,45$ & $192,95 \pm 98,43$ & 0,001 \\
\hline $\log T G$ & $2,16 \pm 0,22$ & $2,23 \pm 0,19$ & 0,010 \\
\hline HDL-C (mg/dll $)$ & $52,51 \pm 13,25$ & $46,66 \pm 13,59$ & 0,002 \\
\hline LDL-C (mg/dL) & $117,02 \pm 33,20$ & $122,23 \pm 31,74$ & 0,258 \\
\hline LDL/HDL & $2,36 \pm 0,84$ & $2,76 \pm 0,91$ & 0,001 \\
\hline Não HDL-C & $144,80 \pm 41,28$ & $152,67 \pm 36,54$ & 0,154 \\
\hline $\mathrm{CT} / \mathrm{HDL}$ & $3,96 \pm 1,20$ & $4,50 \pm 1,26$ & 0,003 \\
\hline TG/HDL & $3,62 \pm 2,73$ & $4,65 \pm 3,21$ & 0,017 \\
\hline Glicemia (mg/dL) & $89,36 \pm 7,78$ & $89,18 \pm 8,91$ & 0,877 \\
\hline Proteína C reativa (mg/dL) & $2,37 \pm 2,06$ & $3,87 \pm 2,85$ & 0,011 \\
\hline Tabagismo & $1,83 \pm 0,38$ & $1,67 \pm 0,51$ & 0,491 \\
\hline
\end{tabular}

Os valores são expressos como $\pm D P$ médio ou porcentagens de valore categóricos. O teste $t$ Student não pareado e o teste qui-quadrado foram usados. IMC: índice de massa corporal; PSS; Pressão sanguínea sistólica; PSD: Pressão sanguínea diastólica; EIMC: espessura da íntima-média da artéria carótida; EIM máx.: Espessura intima-média máxima; HDL-C: Lipoproteína de alta densidade; LDL-C: Lipoproteína de baixa densidade.

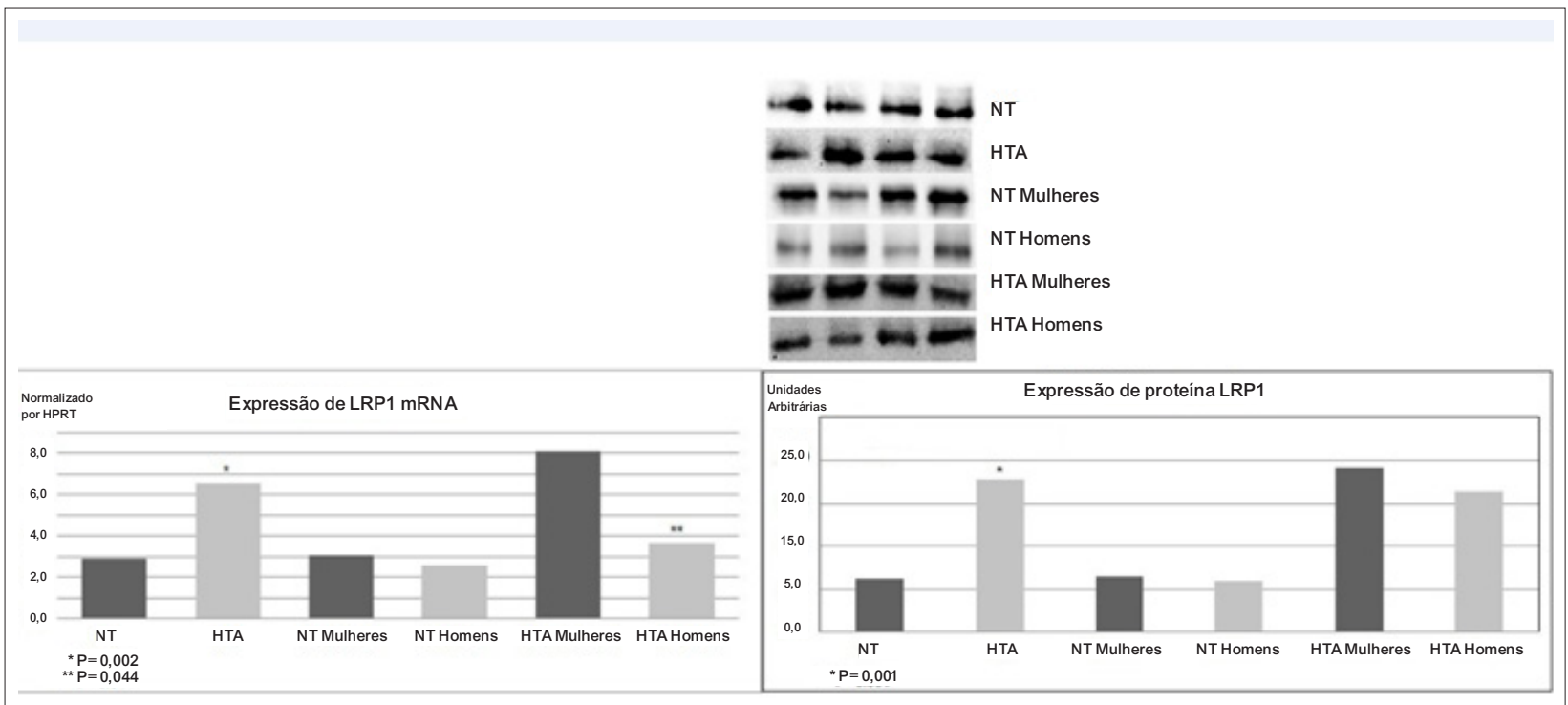

Figura 1 - Quantificação da expressão de LRP1 no total de indivíduos e dividida por sexo. (A) Comparação da expressão de LRP1 em monócitos de indivíduos normotensos e hipertensos. Análise de PCR em tempo real da expressão de LRP1 mRNA. Os dados foram processados com um software especialmente projetado, baseado no valor Ct de cada amostra e normalizado por análise western blot HPRT1 (B) mostrando a expressão de proteína LRP1 em monócitos. 
Tabela 2 - Valores de EIMC e Ang II divididos por sexo

\begin{tabular}{lccccccccc}
\hline & NT & HTA & $\mathbf{p}$ & $\begin{array}{c}\text { NT } \\
\text { Mulheres }\end{array}$ & $\begin{array}{c}\text { NT } \\
\text { Homens }\end{array}$ & $\mathbf{p}$ & $\begin{array}{c}\text { HTA } \\
\text { Mulheres }\end{array}$ & $\begin{array}{c}\text { HTA } \\
\text { Homens }\end{array}$ & $\begin{array}{c}p \\
\text { EIM (mm) }\end{array}$ \\
\hline Ang II (pmol/ml) & $0,568 \pm 0,16$ & $0,715 \pm 0,16$ & 0,002 & $0,553 \pm 0,149$ & $0,583 \pm 0,178$ & 0,303 & $0,692 \pm 0,14$ & $0,719 \pm 0,19$ & 0,643 \\
\hline
\end{tabular}

NT: Normotensos; HTA: Hipertensos; EIM: espessura da intima-média, Ang II: Angiotensina II. Teste t Student não pareado.

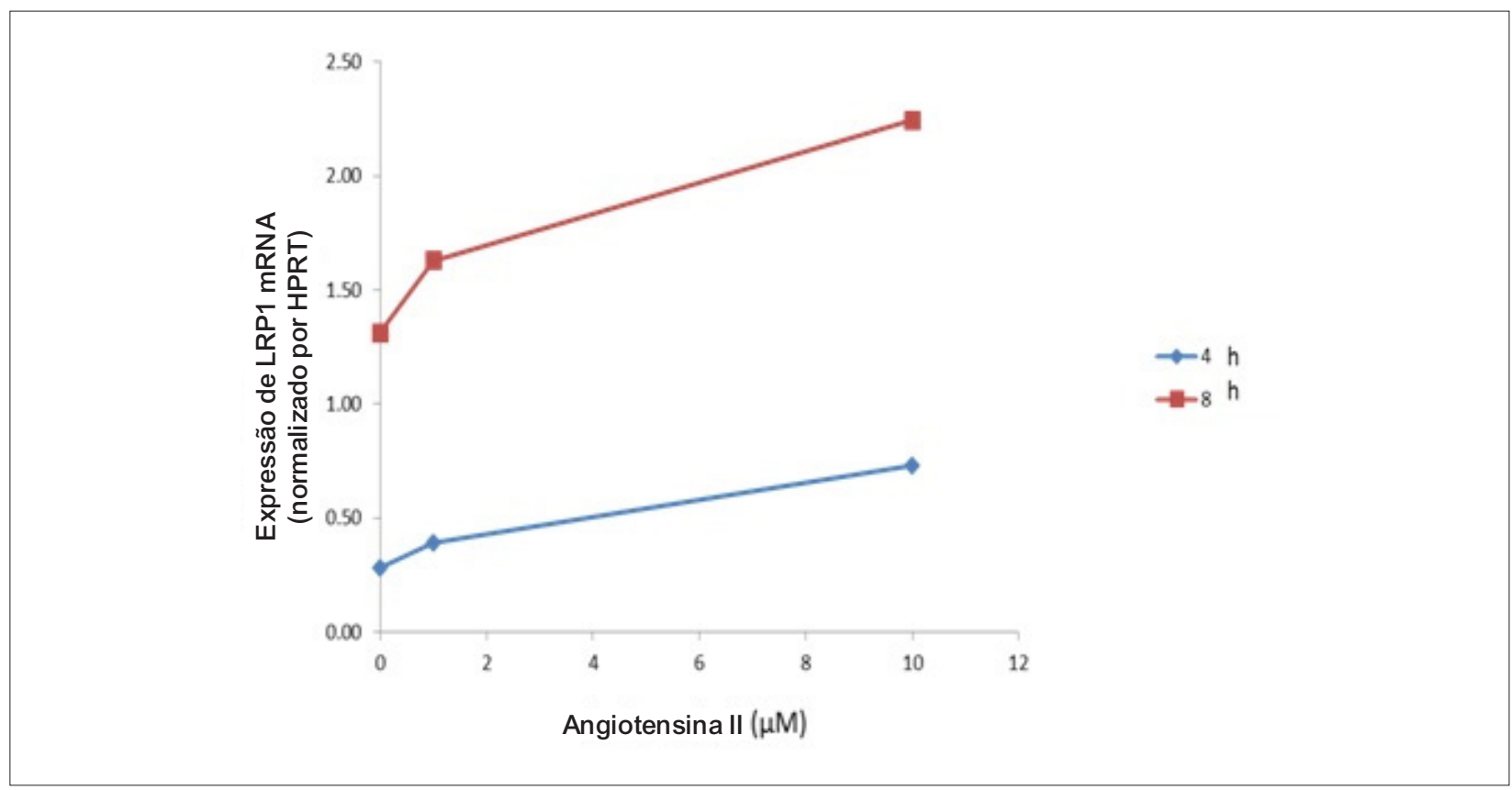

Figura 2 - Efeitos da angiotensina II na expressão de LRP1 em células THP1.

Portanto, uma regressão logística ajustada para lipídios foi realizada para analisar se a dislipidemia poderia influenciar a associação entre LRP1 e EIMC em pacientes hipertensos, (Tabela 3, Modelos 1-4). Foi encontrada entre EIMC e expressão de LRP1 mRNA com todo o conjunto de parâmetros de lipídios: Modelo $1(p=0,045)$, a associação foi mantida após o ajuste de cada parâmetro lipídico: Modelo 2: ajustado por colesterol total $(p=0,053)$, Modelo 3 ajustado por triglicérides $(p=0,049)$, Modelo 4 ajustado por HDL-C $(p=0,038)$, e Modelo 5 ajustado por LDL-C $(p=0,052)$.

Entretanto, não observamos uma associação entre EIMC expressão de proteína LRP1 quando ajustamos o conjunto completo de parâmetros lipídicos, Modelo $1(\mathrm{P}=0,066)$. Entretanto, quando ajustamos cada parâmetro lipídico, encontramos uma associação Modelo 2: ajustado por colesterol total $(p=0,040)$, Modelo 3 ajustado por triglicérides $(p=0,041)$, Modelo 4 ajustado por HDL-C $(p=0,038)$, e Modelo 5 ajustado por LDL-C $(p=0,040)$.

Depois disso, fizemos uma regressão linear entre EIMC e níveis de expressão ajustados por perfil lipídico e expressão de proteína ajustada por perfil lipídico, uma correlação positiva entre essas variáveis foi mantida (Figura 3).

\section{Discussão}

Nossos resultados mostraram, conforme esperado, que a EIMC média era mais alta nos indivíduos hipertensos. Entretanto, esse valor foi associado de maneira importante com a expressão excessiva de LRP1 nos monócitos circulantes.

A EIMC é considerada um marcador de aterosclerose e um indicador excelente de morte e eventos cardiovasculares. ${ }^{26}$ Em pacientes hipertensos com doença arterial coronariana, a EIMC aumentada está intimamente associada a aterosclerose. ${ }^{27}$ Nossos dados mostram uma associação forte entre hipertensão e EIMC. Esses resultados estão de acordo com dados publicados anteriormente em estudos realizados em pacientes e modelos animais. Em um estudo envolvendo jovens com hipertensão limítrofe (130-140/85-89 mmHg), observou-se um aumento de EIMC nas artérias braquiais quando os pacientes foram comparados com sujeitos normotensos; foram encontradas

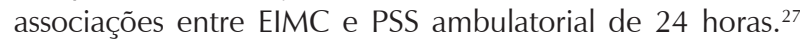
Além disso, hipertensão, juntamente com diabetes e idade, são consideradas fatores prognósticos independentes para hiperplasia da íntima na artéria radial. ${ }^{28-30}$ In Em um modelo de animais com hipertensão, foi relatado um espessamento significativo da íntima-média como causa direta da doença. ${ }^{31,32}$ 


\section{Artigo Original}

Tabela 3 - Associação entre a expressão de LRP1 e EIMC ajustada para parâmetros lipídicos em pacientes com hipertensão

\begin{tabular}{lcc}
\hline mRNA & RC [95\% de IC] & $\mathbf{p}$ \\
\hline Ajuste [-] & $0,308[0,230-38,650]$ & 0,047 \\
\hline Modelo 1 & $0,310[0,340-38,887]$ & 0,046 \\
\hline Modelo 2 & $0,303[-0,280-38,511]$ & 0,053 \\
\hline Modelo 3 & $0,308[0,131-38,832]$ & 0,049 \\
\hline Modelo 4 & $0,312[0,150-38,33]$ & 0,038 \\
\hline Modelo 5 & $0,301[-0,181-38,19]$ & 0,052 \\
\hline Proteína & $\mathrm{RC}[95 \%$ de IC] & $\mathbf{p}$ \\
\hline ajuste [-] & $0,312[1,771-65,319]$ & 0,039 \\
\hline Modelo 1 & $0,294[-2,150-65,208]$ & 0,066 \\
\hline Modelo 2 & $0,211[1,544-65,637]$ & 0,040 \\
\hline Modelo 3 & $0,313[1,445-65,77]$ & 0,041 \\
\hline Modelo 4 & $0,317[2,020-66,015]$ & 0,038 \\
\hline Modelo 5 & $0,313[1,528-65,6689]$ & 0,040 \\
\hline
\end{tabular}

Modelo 1: ajustado por todos os parâmetros lipídicos. Modelo 2 ajustado por colesterol total. Modelo 3: ajustado por triglicérides Modelo 4: ajustado por HDL-C. Modelo 5: ajustado por LDL-C. Análise de regressões logística múltipla.
A hipertensão está entre os principais fatores de risco na etiologia da doença vascular aterosclerótica. ${ }^{33,34}$ Entretanto os mecanismos pelos quais a pressão arterial aumenta a incidência de aterosclerose não estão totalmente claros. Estudos que focam em elucidar esses mecanismos têm uma importância crítica. Há uma associação forte entre hipertensão e a expressão de LRP1 na parede vascular de um modelo com rato. ${ }^{35}$ A upregulation da LRP1 por hipertensão tem consequências funcionais, já que promove acúmulo de lipídios intracelular e, portanto, a formação de células espumosas. A hipertensão também tem um impacto alto na remodelagem vascular, mudanças crônicas em forças hemodinâmicas, e alterações estruturais na parede vascular. ${ }^{36}$

Nossos resultados mostram a exposição excessiva em mRNA e expressão de receptor de LRP1 em monócitos de pacientes hipertensos. Nossos resultados também mostram que Ang II aumentou a expressão de LRP1 em culturas de THP-1 de maneira dependente de dose e tempo. Portanto, o mecanismo pelo qual a pressão sanguínea alta poderia regular a expressão de LRP1 poderia ser mediado pelo efeito da angiotensina II, que é considerado um dos principais mediadores de hipertensão. Também se relatou que a angiotensina induz a atividade dos fatores de transcrição $\mathrm{Sp} /$ Sp3, que estão envolvidos no reconhecimento do promotor LRP $1{ }^{13}$ causando a expressão excessiva em um nível vascular e favorecendo a formação de células espumosas nas células musculares lisas vasculares humanas. ${ }^{33}$
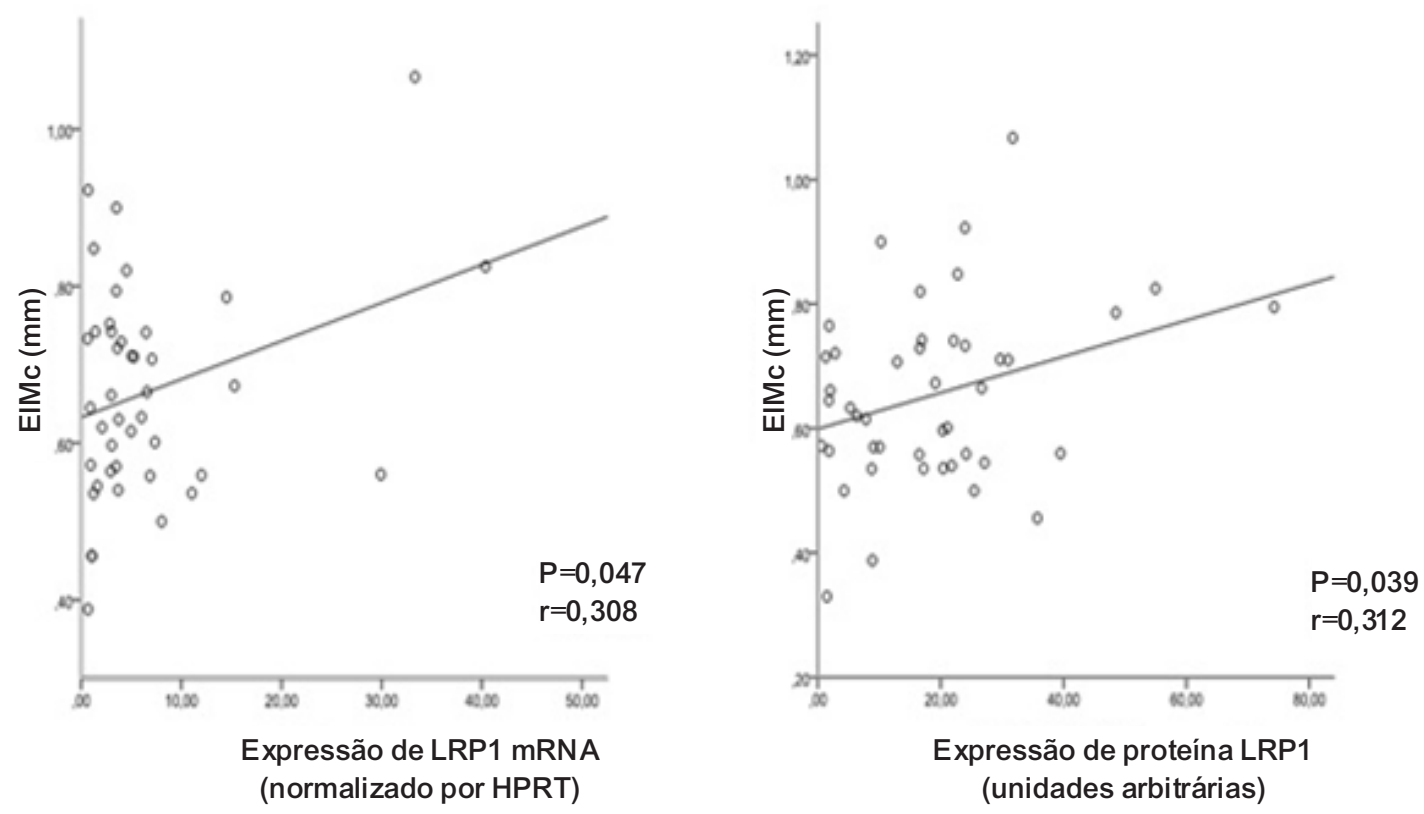

Figura 3 - Correlação entre EIM e niveis de expressão de mRNA e proteina LRP1 ajustada por CT, TG, HDL-C e LDL-C. P<0,005 é considerado estatisticamente significativo. 
Além da angiotensina II, o fluxo sanguíneo age na função e na estrutura do endotélio pela modulação da expressão gênica. ${ }^{37}$ As alterações funcionais que são sofridas pelos monócitos devido às contínuas mudanças em fluxo sanguíneo podem ter uma influência positiva na expressão do LRP1, estimulando a captação de LDL e causando um aumento na EIMC.

Além de uma alta prevalência da obesidade, a população mexicana está enfrentando um problema sério de dislipidemia, que foi explicada por uma interação de fatores genéticos e ambientais. ${ }^{38}$

$\mathrm{Na}$ análise de sujeitos com dislipidemia de acordo com o risco cardiovascular convencional, observamos um aumento na expressão de LRP1 mRNA e de proteína em indivíduos dislipidêmicos hipertensos, o que poderia significar que LRP1 tem expressão excessiva por hipertensão independente da dislipidemia.

Estudos prévios mostraram que a concentração de proteína-1 relacionada a receptor de lipoproteína de baixa densidade circulante solúvel (sLRP1) poderia estar intimamente associado a hipercolesterolemia (LDL-C $>200$ $\mathrm{mg} / \mathrm{dL}$ ) e um efeito de upregulation de hipercolesterolemia na expressão de LRP1 em células dos modelos in vitro e in vivo da parede vascular. ${ }^{39} \mathrm{Em}$ nossos resultados, apesar de observarmos uma porcentagem alta de hipercolesterolemia em indivíduos normotensos e hipertensos, não foram encontradas diferenças significativas entre ambos os grupos. Uma explicação possível sobre essas diferenças poderia ser: a) a associação entre sLPR1 e colesterol foi realizada em populações hipercolesterolêmicas (com hipercolesterolemia grave); b) a LRP1 poderia ser expressa em uma variedade de tecidos e a especificidade poderia ser diferente; em nosso caso, medimos a expressão de LRP1 em monócitos; c) as populações são muito diferentes, nosso estudo é feito em uma mistura de indígenas americanos [65\%], europeus [31\%], e africanos [3\%], enquanto o deles foi feito inteiramente com caucasianos. ${ }^{40}$

Nossos dados indicam que a expressão de LRP1 em monócitos de pacientes hipertensos está correlacionada com o aumento de EIMC. A regressão logística ajustada mostra que a correlação entre EIMC e expressão de LRP1 mRNA é mantida mesmo após o ajuste de parâmetros lipídicos. Entretanto, essa associação foi perdida quando o ajuste foi feito com proteína LRP1. Esses resultados podem ser explicados pelo efeito positivo forte de LDL modificado sobre a estabilidade da proteína LRP1. ${ }^{41,42}$ Portanto, a dislipidemia provavelmente contribui para manter uma expressão de proteína LRP1 em monócitos alta em pacientes hipertensos. Isso poderia justificar porque a associação entre EIMC e expressão de proteína LRP1 após o ajuste do perfil lipídico é perdida.

\section{Conclusões}

Nossos achados permitem que sugiramos que o efeito da hipertensão na aterosclerose pode ocorrer pela expressão excessiva de LRP1 em monócitos circulantes. A Ang II induziu a upregulation de LRP1 em monócitos, e pode ter um papel importante no aumento de EIMC associado ao fator de risCo cardiovascular indução de progressão de lesão aterosclerótica. Esses resultados reforçam que a alta relevância de expressão excessiva de LRP1 na formação e na progressão de placas ateroscleróticas em seres humanos.

\section{Contribuição dos Autores}

Concepção e desenho da pesquisa: Llorente-Cortés VC, Huesca-Gómez C; Obtenção de dados: Gamboa R, Jaramillo-Estrella MJ, Martínez-Alvarado M del R, Torres-Paz YE, Gonzalo-Calvo D, Del Valle-Mondragón L, López-Marure R; Análise e interpretação dos dados: Gamboa R, JaramilloEstrella MJ, Soto ME, Huesca-Gómez C; Análise estatística: Soto ME, Huesca-Gómez C; Obtenção de financiamento: Huesca-Gómez C; Redação do manuscrito: Gamboa R, Jaramillo-Estrella MJ, Llorente-Cortés VC, Huesca-Gómez C; Revisão crítica do manuscrito quanto ao conteúdo intelectual importante: Gamboa R, Llorente-Cortés VC.

\section{Potencial Conflito de Interesses}

Declaro não haver conflito de interesses pertinentes.

\section{Fontes de Financiamento}

O presente estudo foi financiado pelo Apoio Complementar para Pesquisadores em Processo de Consolidação SNI-1-2009 de CONACyT número 119410.

\section{Vinculação Acadêmica}

Este artigo é parte de dissertação de Mestrado de María José Jaramillo-Estrella pela Universidad Nacional Autonoma de México. 


\section{Referências}

1. Bhadoria AS, Kasar PK, Toppo NA, Bhadoria P, Pradhan S, Kabirpanthi V. Prevalence of hypertension and associated cardiovascular risk factors in Central India. J Family Community Med. 2014;21(1):29-38.

2. Solberg LA, Strong JP. Risk factors and atherosclerotic lesions. A review of autopsy studies. Arteriosclerosis, 1983;3(3):187-98.

3. Hoffman RP. Vascular endothelial dysfunction and nutritional compounds in early type 1 diabetes. Curr Diabetes Rev. 2014;10(3):201-7.

4. Meljarejo E. Memorias: el papel del óxido nítrico en la enfermedad aterosclerótica. Act Med Colomb. 2001;26(4):200-1.

5. Badimon L, Vilahur G, Padró T. Lipoproteins, platelets and atherothrombosis. Rev Esp Cardiol. 2009;62(10):1161-78.

6. Luoma J, Hiltunen T, Särkioja T, Moestrup SK, Gliemann J, Kodama T, et al. Expression of alpha 2-macroglobulin receptor/low density lipoprotein receptor-related protein and scavenger receptor in human atherosclerotic lesions. J Clin Invest. 1994;93(5):2014-21.

7. Gonias SL, Campana WM. LDL receptor-related protein-1: a regulator of inflammation in atherosclerosis, cancer, and injury to the nervous system. Am J Pathol. 2014;184(1):18-27.

8. Ferrer DG, Jaldin-Fincati JR, Amigone JL, Capra RH, Collino, CJ, Albertini RA, et al. Standardized flow cytometry assay for identification of human monocytic heterogeneity and LRP1 expression in monocyte subpopulations: decreased expression of this receptor in non classical monocytes. Cytom. Part A. 2014;85(7): 601-10.

9. Handschug K, Schulz S, Schnürer C, Köhler S, Wenzel K, Teichmann W, et al. Low-density lipoprotein receptor-related protein in atherosclerosis development: up-regulation of gene expression in patients with coronary obstruction. J Mol Med (Berl). 1998;76(8):596-600.

10. Hiltunen TP, Luoma JS, Nikkari T, Ylä-Herttuala S: Expression of LDL receptor, VLDL receptor, LDL receptor-related protein, and scavenger receptor in rabbit atherosclerotic lesions: marked induction of scavenger receptor and VLDL receptor expression during lesion development. Circulation. 1998;97(11):1079-86.

11. Llorente-Cortes V, Otero-Viñas M, Berrozpe M, Badimon L. Intracellular lipid accumulation, low-density lipoprotein receptor-related protein expression, and cell survival in vascular smooth muscle cells derived from normal and atherosclerotic human coronaries. Eur J Clin Invest. 2004;34(3):182-90.

12. Schulz S, Birkenmeier G, Schagdarsurengin U, Wenzel K, Müller-Werdan $U$, Rehfeld D, et al. Role of LDL receptor-related protein (LRP) in coronary atherosclerosis. Int J Cardiol. 2003;92(2-3):137-44.

13. Schulz S, Schagdarsurengin U, Greiser P, Birkenmeier G, Müller-Werdan $U$, Hagemann M, et al. The LDL receptor-related protein (LRP1/A2MR) and coronary atherosclerosis--novel genomic variants and functional consequences. Hum Mutat. 2002;20(5):404

14. Llorente-Cortés V, Royo T, Otero-Viñas M, Berrozpe M, Badimon L. Sterol regulatory element binding proteins downregulate $\mathrm{LDL}$ receptor-related protein (LRP1) expression and LRP1-mediated aggregated LDL uptake by human macrophages. Cardiovasc Res. 2007;74(3):526-36.

15. Sakr SW, Eddy RJ, Barth H, Wang F, Greenberg S, Maxfield FR, et al. The uptake and degradation of matrix-bound lipoproteins by macrophages require an intact actin Cytoskeleton, Rho family GTPases, and myosin ATPase activity. J Biol Chem. 2001;276(40):37649-58.

16. Llorente-Cortés V, Gonzalo-Calvo D, Orbe J, Páramo JA, Badimon L. Signature of subclinical femoral artery atherosclerosis in peripheral blood mononuclear cells. Eur J Clin Invest. 2014;44(6):539-48.

17. Halcox JP, Donald AE, Ellins E, Witte DR, Shipley MJ, Brunner EJ, et al. Endothelial function predicts progression of carotid intima-media thickness. Circulation. 2009;119(7):1005-12.
18. Lind L, Andersson J, Rönn M, Gustavsson T, Holdfelt P, Hulthe J, et al. Brachial artery intima-media thickness and echogenicity in relation to lipids and markers of oxidative stress in elderly subjects:-the prospective investigation of the vasculature in Uppsala Seniors (PIVUS) Study. Lipids. 2008;43(2):133-41.

19. Koyoshi R, Miura S, Kumagai N, Shiga Y, Mitsutake R, Saku K. Clinical significance of flow-mediated dilation, brachial intima-media thickness and pulse wave velocity in patients with and without coronary artery disease. Circ J. 2012;76(6):1469-75.

20. Laurent S, Boutouyrie P, Asmar R, Gautier I, Laloux B, Guize L, et al. Aortic stiffness is an independent predictor of all-cause and cardiovascular mortality in hypertensive patients. Hypertension. 2001;37(5):1236-41.

21. Hafner F, Kieninger A, Meinitzer A, Gary T, Froehlich H, Haas E, et al. Endothelial dysfunction and brachial intima-media thickness: long term cardiovascular risk with claudication related to peripheral arterial disease: a prospective analysis. PLoS One. 2014;9(4):e93357.

22. Stein JH, Korcarz CE, Hurst RT, Lonn E, Kendall CB, Mohler ER, et al. Use of carotid ultrasound to identify subclinical vascular disease and evaluate cardiovascular disease risk: a consensus statement from the American Society of Echocardiography Carotid Intima-Media Thickness Task Force Endorsed by the Society for Vascular Medicine. J Am Soc Echocardiography. 2008;21(2):93-111.

23. DeLong DM, DeLong ER, Wood PD, Lippel K, Rifkind BM. A comparison of methods for the estimation of plasma low-and very low-density lipoprotein cholesterol. The Lipid Research Clinics Prevalence Study. JAMA. $1986 ; 256(17): 2372-7$

24. Tenorio-López FA, Zarco-Olvera G, Sánchez-Mendoza A, Rosas-Peralta M, Pastelín-Hernández G, Valle-Mondragón L. Simultaneous determination of angiotensins II and 1-7 by capillary zone electrophoresis in plasma and urine from hypertensive rats. Talanta. 2010;80(5):1702-12.

25. Sendra J, Llorente-Cortés V, Costales P, Huesca-Gómez C, Badimon L. Angiotensin II upregulates LDL receptor-related protein (LRP1) expression in the vascular wall: a new pro-atherogenic mechanism of hypertension. Cardiovasc Res. 2008;78(3):581-9.

26. Zielinski T, Dzielinska Z, Januszewicz A, Rynkun D, Makowiecka Ciesla $M$, Tyczynski $P$, et al. Carotid intima-media thickness as a marker of cardiovascular risk in hypertensive patients with coronary artery disease. Am J Hypertens. 2007;20(10):1058-64.

27. Amato M, Montorsi P, Ravani A, Oldani E, Galli S, Ravagnani PM, et al. Carotid intima-media thickness by B-mode ultrasound as surrogate of coronary atherosclerosis: correlation with quantitative coronary angiography and coronary intravascular ultrasound findings. Eur Heart J. 2007;28(17):2094-101.

28. Toikka JO, Laine H, Ahotupa M, Haapanen A, Viikari JS, Hartiala JJ, et al. Increased arterial intima-media thickness and in vivo LDL oxidation in young men with borderline hypertension. Hypertension. 2000;36(6):929-33

29. Chowdhury UK, Airan B, Mishra PK, Kothari SS, Subramaniam GK, Ray R, et al. Histopathology and morphometry of radial artery conduits: basic study and clinical application. Ann Thorac Surg. 2004;78(5):1614-21.

30. Ruengsakulrach P, Sinclair R, Komeda M, Raman J, Gordon I, Buxton B. Comparative histopathology of radial artery versus internal thoracic artery and risk factors for development of intimal hyperplasia and atherosclerosis. Circulation. 1999;100(19 Suppl):II139-44.

31. Kowala MC, Cuénoud HF, Joris I, Majno G. Cellular changes during hypertension: a quantitative study of the rat aorta. Exp Mol Pathol. 1986;45(3):323-35

32. Rossi MA, Colombini-Netto M. Chronic inhibition of NO synthesis per se promotes structural intimal remodeling of the rat aorta. J Hypertens. 2001;19(9):1567-79.

33. Simon A, Levenson J. Stratification of vascular risk in hypertension and therapeutic perspective. Am J Hypertens. 1995;8(10 Pt 2):45S-48. 
34. Violi F, Criqui M, Longoni A, Castiglioni C. Relation between risk factors and cardiovascular complications in patients with peripheral vascular disease. Results from the A.D.E.P. study. Atherosclerosis. 1996;120(1):25-35.

35. Aledo R, Costales P, Ciudad C, Noé V, Llorente-Cortes V, Badimon L. Molecular and functional characterization of LRP1 promoter polymorphism c.1-25 C>G (rs138854007). Atherosclerosis. 2014;233(1):178-85.

36. Baumbach GL, Heistad DD. Remodeling of cerebral arterioles in chronic hypertension. Hypertension. 1989;13(6 Pt 2):968-72.

37. Davies PF, Tripathi SC. Mechanical stress mechanisms and the cell. An endothelial paradigm. Circ Res. 1993;72(2):239-45.

38. Aguilar-Salinas CA, Gómez-Pérez FJ, Rull J, Villalpando S, Barquera S, Rojas R. Prevalence of dyslipidemias in the Mexican National Health and Nutrition Survey 2006. Salud Pública Mex. 2010;52(Suppl 1):S44-53.
39. Gonzalo-Calvo D, Cenarro A, Martinez-Bujidos M, Badimon L, Bayes-Genis A, Ordonez-Llanos J, et al. Circulating soluble low-density lipoprotein receptor- related protein 1 (SLRP1) concentration is associated with hipercolesterolemia: a new potential biomarker for atherosclerosis. Int ] Cardiol. 2015 Dec 15;201:20-9.

40. Johnson NA, Coram NA, Shriver MD, Romieu I, Barsh GS, London SJ, et al. Ancestral components of admixed genomes in a Mexican cohort. PloS Genet. 2011;7(12):e1002410.

41. Llorente-Cortes V, Casani L, Cal R, Llenas A, Juan-Babot O, Camino-López $\mathrm{S}$, et al. Cholesterol-lowering strategies reduce vascular LRP1 overexpression induced by hypercholesterolaemia. Eur J Clin Invest. 2011;41(10):1087-97.

42. Cal R, García-Arguinzonis M, Revuelta-López E, Castellano J, Padró T, Badimon L, et al. Aggregated low-density lipoprotein induces LRP1 stabilization through E3 ubiquitin ligase CHFR downregulation in human vascular smooth muscle cells. Arterioscler Thromb Vasc Biol. $2013 \cdot 33(2): 369-77$ 